\title{
ON THE REDUCTION OF \\ PERMUTATION REPRESENTATIONS
}

\section{B. M. Puttaswamaiah}

(received October 1, 1962)

Introduction. In this paper an attempt is made to generalize the rather special role played by certain "Young subgroups" of $\mathrm{S}_{\mathrm{n}}$. In Theorem 1, the substitutional equation involving Young's raising operators for $S_{n}$ is generalized to apply to an arbitrary subgroup $\mathrm{H}$ of a finite group $\mathrm{G}$. Applying this to $S_{n}$, it is shown how the multiplicities $\mathrm{m}_{\mathrm{H}}^{\lambda}$ can be calculated in terms of the raising operators in Theorem 2, which calculations are illustrated by a simple example.

The second part of the paper considers the converse problem of constructing a basis of permutation representations for the irreducible representations. The permutation character $\Phi_{i}^{H}$ of a conjugate class $C_{i}$, containing $g_{i}$ elements, in a permutation representation $\mathrm{G}^{\mathrm{H}}$ of $\mathrm{G}$ of order $\mathrm{g}$ (induced by the identity representation of $\mathrm{H}$ of order $\mathrm{h}$ ) is

$$
\Phi_{i}^{H}=\frac{g_{i}^{H}}{h g_{i}}
$$

where $g_{i}^{H}$ is the number of elements of $C_{i}$ in $H$. A basis for an irreducible representation of $G$ in terms of permutation representation is possible only if all the irreducible characters are rational. This is applied to generalize Young' s definition of the idempotents of the group algebra in Theorem 3, without

Canad. Math. Bull. vol. 6, no. 3, September 1963 
the explicit use of characters. An illustrative example makes all this clear.

Finally I would express my thanks to Professor G. de B. Robinson for his help in writing this article.

1. Let $G$ be a finite group of order $g$ and $H$ be a subgroup of order $h$. Let $C_{1}, C_{2}, \ldots, C_{k}$ be the $k$ conjugate classes of G, as well as the algebraic sums of the elements of the classes. The number of elements in $C_{i}$ will be denoted by $g_{i}$. If the algebraic sum of the elements of the subgroup $\mathrm{H}$ is denoted by $\mathrm{P}^{\mathrm{H}}$, we denote by $\Sigma i \mathrm{P}^{\mathrm{H}}$ the algebraic sum $\sum_{t=1}^{g} A_{t}^{-1} P^{H} A_{t}$ and call it the $\Sigma^{\prime}$ sum of the subgroup $H$ in $G$. Then we have

THEOREM 1. If $\mathrm{H}$ is a subgroup of order $\mathrm{h}$ of the finite group $G$ of order $g$ and $\Sigma^{\prime} P^{H}$ is the $\Sigma^{\prime}$ sum of $H$, then

1.1

$$
\Sigma \cdot P^{H}=\underset{\rho}{h}{\underset{m}{H}}^{\rho} S^{\rho},
$$

where the summation runs over all the irreducible representations $\rho$ of $G$ over the complex field, $\mathrm{m}_{\mathrm{H}}{ }^{\rho}$ is the multiplicity of the irreducible representation $\rho$ in the permutation representation of $G$ induced by the identity representation of $\mathrm{H}$, and $\mathrm{f}^{\rho} \mathrm{S}^{\rho} / \mathrm{g}$ is the idempotent in the group algebra associated with the irreducible representation $\rho$ of degree $f^{P}$.

Proof. It is well known that the idempotents $L^{p}$ are obtained by setting

$$
L^{\rho}=\frac{f^{\rho}}{g} S^{\rho} \text { where } S^{\rho}=\sum_{i=1}^{k} x_{i}{ }^{\rho} C_{i^{\prime}}
$$


and $X_{i^{r}}{ }^{\rho}$ is the character of the class $C_{i}$ in the irreducible representation $\rho$ of $G . \quad C_{i^{\prime}}$ is the inverse class of $C_{i}$.

If $\Phi_{i}^{H}$ is the permutation character of the class $C_{i}$ in the representation of $G$ induced by the identity representation of $\mathrm{H}$, we can write

1. 3

$$
\Phi_{i}^{H}=\frac{g_{i}^{H}}{h g_{i}}=\Sigma m_{\rho} \rho x_{i^{\prime}}^{\rho} \text {, }
$$

where $g_{i}{ }^{H}$ is the number of elements of the class $C_{i}$ which lie in $\mathrm{H}$.

If $A_{i}$ is any element of the class $C_{i}$ and the element $A_{t}$ runs through the $g$ elements of $G$, then the $g$ elements $A_{t}^{-1} A_{i} A_{t}$ consist of the conjugate class $C_{i}$ counted $g / g_{i}$ times, i.e.

1. 4

$$
\sum_{t=1}^{g} A_{t}^{-1} A_{i} A_{t}=\frac{g}{g_{i}} C_{i} .
$$

Since the number of elements of the class $C_{i^{\prime}}$ which lie in $H$ is also, $g_{i}^{H}$, we have

$$
\begin{aligned}
& \Sigma^{\prime} P^{H}=\sum_{i=1}^{k} \frac{g g_{i}^{H}}{g_{i}} C_{i^{\prime}} \\
&=h \sum_{i=1}^{k} \Phi_{i}^{H} C_{i^{\prime}} \\
&=h \sum_{H^{\rho}}^{\rho}{ }^{j} x_{i}^{\rho} C_{i^{\prime}} \\
&=h \sum m_{H}^{\rho} S^{\rho}, \\
& \rho
\end{aligned}
$$


as desired.

The result 1.1 is a generalization of Young's substitutioral equation for certain subgroups of the symmetric group $S_{n}$, which are themselves products of symmetric groups; we call these "Young subgroups" in the sequel. In the case of $\mathrm{S}_{n}$, a method of constructing the idempotents without characters has been given by Young [3]. If $Y$ is the Young symmetrizer of the diagram $[\lambda]$ associated with the partition $\left(\lambda_{1} \lambda_{2} \lambda_{3} \ldots \lambda_{h}\right)$ of $n$, then

$$
\mathrm{S}^{\lambda}=\frac{\mathrm{f}^{\lambda}}{\mathrm{n} !} \mathrm{\sigma}_{\sigma}^{-1} \mathrm{Y} \sigma
$$

where the summation runs over all elements $\sigma$ of $S_{n}$, so tha the above theorem reads: If $[\lambda]$ is an irreducible representat $n$ of $S_{n}$, associated with the partition $(\lambda)$ of $n$, then

$$
\frac{1}{h} \Sigma^{\prime} \mathrm{P}^{\mathrm{H}}=\sum_{\lambda} \mathrm{m}_{\mathrm{H}}{ }^{\lambda} \mathrm{S}^{\lambda}
$$

If $H$ be a Young subgroup, then

1.5

$$
\left[\lambda_{1}\right] \cdot\left[\lambda_{2}\right] \ldots\left[\lambda_{h}\right]=\prod_{i, j}\left(1-R_{i j}\right)^{-1}\left[\lambda_{1} \lambda_{2} \ldots \lambda_{h}\right]
$$

where $\left[\lambda_{1}\right] \cdot\left[\lambda_{2}\right] \ldots\left[\lambda_{h}\right]$ is the permutation representation of $S_{n}$ induced by the identity representation of the Young subgroup $\mathrm{H}=\mathrm{S}_{\lambda_{1}} \times \mathrm{S}_{\lambda_{2}} \times \ldots \times \mathrm{S}_{\lambda_{\mathrm{h}}},\left[\lambda_{1} \lambda_{2} \cdots \lambda_{h}\right]$ is the irreducible representation of $S_{n}$ associated with the Young diagram $[\lambda]$, and $R_{i j}$ is Young's raising operator with the usual restrictions [3].

The knowledge of the reduction of the permutation wepresentation of $\mathrm{S}_{\mathrm{n}}$ induced by such a Young subgroup may be used in the reduction of the representation induced by any 
arbitrary subgroup $\mathrm{H}$ of $\mathrm{S}_{\mathrm{n}}$; thus:

THEOREM 2. If $H$ is any subgroup of order $h$ of $S_{n}$, then the problem of reducing the permutation representation of

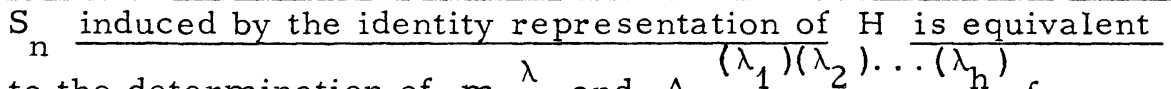
to the determination of $\mathrm{m}_{\mathrm{H}}^{\lambda}$ and $\mathrm{A}_{\mathrm{H}}\left(\lambda_{1}\right)\left(\lambda_{2}\right) \ldots\left(\lambda_{\mathrm{h}}\right)$ from

$$
(I) H \uparrow S_{n}=\sum_{\left[1^{n}\right]}^{[n]} m_{H} \lambda[\lambda] \text { and } h m_{H}^{\lambda}=\underset{i j}{\lambda}\left(1-R_{i j}\right) A_{H}\left(\lambda_{1}\right)\left(\lambda_{2}\right) \ldots\left(\lambda_{h}\right)
$$

where

$$
A_{H}\left(\lambda_{1}\right)\left(\lambda_{2}\right) \ldots\left(\lambda_{h}\right)=\sum_{t=1}^{k} g_{t} H_{t}\left(\lambda_{1}\right)\left(\lambda_{2}\right) \ldots\left(\lambda_{h}\right)
$$

and the raising operators act on the index of $\mathrm{A}_{\mathrm{H}}$

$$
\left(\lambda_{1}\right)\left(\lambda_{2}\right) \ldots\left(\lambda_{h}\right)
$$

just as for the permutation representation.

Proof. From 1.5, we write

1.6

$$
x_{t}^{\lambda}=\prod_{i j}\left(1-R_{i j}\right) \Phi_{t}^{\left(\lambda_{1}\right)\left(\lambda_{2}\right) \cdots\left(\lambda_{h}\right)}
$$

where $\Phi_{t}$

$$
\left(\lambda_{1}\right)\left(\lambda_{2}\right) \ldots\left(\lambda_{h}\right)
$$

$t$-th class in $\left[\lambda_{1}\right] \cdot\left[\lambda_{2}\right] \ldots\left[\lambda_{h}\right]$. If $H$ is of order $h$ the equation 1.3 may be solved for the $\mathrm{m}_{\mathrm{H}}{ }^{\lambda}$ to yield

$$
\begin{aligned}
m_{H}^{\lambda} & =1 / h \sum_{t=1}^{k} g_{t}{ }^{H} x_{t}^{\lambda} \\
& =1 / h \sum_{t=1}^{k} g_{t} \prod_{i, j}^{H}\left(1-R_{i j}\right) \Phi_{t}\left(\lambda_{1}\right)\left(\lambda_{2}\right) \ldots\left(\lambda_{h}\right)
\end{aligned}
$$

which can be written 


$$
h m_{H}^{\lambda}=\prod_{i, j}\left(1-R_{i j}\right) A_{H}\left(\lambda_{1}\right)\left(\lambda_{2}\right) \ldots\left(\lambda_{h}\right),
$$

with

$$
A_{H}\left(\lambda_{1}\right)\left(\lambda_{2}\right) \ldots\left(\lambda_{h}\right)=\sum_{t=1}^{k} g_{t} H_{t}\left(\lambda_{1}\right)\left(\lambda_{2}\right) \ldots\left(\lambda_{h}\right)
$$

as stated above.

Example 1. To clarify the ideas in Theorem 2, consider the alternating group $\mathrm{A}_{3}$ which is not a Young subgroup of $\mathrm{S}_{3}$. Setting $\mathrm{H}=\mathrm{A}_{3}$, the reduction is given by

(I) $\mathrm{A}_{3} \uparrow \mathrm{S}_{3}=\frac{1}{3}\left[1^{3}\right] \Pi\left(1-\mathrm{R}_{\mathrm{ij}}\right) \mathrm{A}_{\mathrm{H}}(1)(1)(1)+\frac{1}{3}[2,1] \Pi\left(1-\mathrm{R}_{\mathrm{ij}}\right) \mathrm{A}_{\mathrm{H}}(2)(1)$

$$
\begin{aligned}
& +\frac{1}{3}[3] \Pi\left(1-R_{i, j}\right) A_{H}(3) \\
=\frac{1}{3}\left[1^{3}\right]\left\{A_{H}(1)(1)(1)-\right. & \left.A_{H}(2)(1)-A_{H}(2)(1)+A_{H}{ }^{(3)}\right\} \\
& +\frac{1}{3}[2,1]\left\{A_{H}(2)(1)-A_{H}(3)\right\} \\
& +\frac{1}{3}[3] \cdot 1 \cdot A_{H}(3) .
\end{aligned}
$$

Since $A_{H}(1)(1)(1)=6, A_{H}{ }^{(2)(1)}=3$ and $A_{H}{ }^{(3)}=3$, we have

$$
\text { (I) } \mathrm{A}_{3} \uparrow \mathrm{S}_{3}=\left[1^{3}\right]+[3]
$$

where $\uparrow$ denotes the inducing process.

2. The converse problem, namely the investigation of properties of the repsentations of $G$ determined by properties of the representations of suitable subgrouns. has heen considered 
by Brauer [1]. He has shown that every character of $G$ can be expressed as a linear combination of the characters of $G$ induced by characters of the (elementary) subgroups. In the following, we shall restrict ourselves to groups whose irreducible characters are all rational, in which case it is only necessary to use permutation representations of $G$, i.e. representations induced by the identity representations of subgroups. For example the symmetric group $S_{n}$ and the hyperoctohedral group $S_{n, 2}$ fall into this category.

It was shown earlier by Prokop [2] that ar irreducible character of $G$ can be expressed as a linear combination with integral coefficients of the characters of $G$ induced by the characters of $k$ (proper) subgroups, if with every conjugate class $C_{j}$ of $G$ there is associated a subgroup $H_{j}$ containing an element of $\mathrm{C}_{j}$, and the indices of the subgroups are relatively prime. The second condition is necessary only to make the coefficients in the linear combination integral. In the present case our coefficients are rational and the following result holds.

THEOREM 3. Let $G$ be a group of finite order $g$, whose irreducible characters are all rational, and $\mathrm{H}_{i}(\mathrm{i}=1,2,3, \ldots, \mathrm{k})$ be a set of $\mathrm{k}$ distinct subgroups of $\mathrm{G}$ such that each subgroup $H_{i}$ contains an element of the class $C_{i}$ If $\rho_{1}, \rho_{2}, \ldots, \rho_{k}$ are the $k$ irreducible representations of $G$, then there exists a non-singular matrix $N=\left(n_{s}{ }^{p}\right)$ of degree $k$, with rational coefficients, such that the expression

$$
L^{\rho}=\frac{f^{\rho}}{g} \sum_{t=1}^{k} n_{t}^{\rho} \frac{\Sigma P^{H}}{h_{t}} .
$$

is the idempotent of the group algebra associated with the irreducible representation $\rho$ of $G$.

Proof. If $\Phi_{i}^{{ }^{t}}$ is the character of the $i-t h$ class $C_{i}$ in the permutation representation of $G$ induced by the identity 
representation of $\mathrm{H}_{t}$, by Prokop's result we can write

2. 1

$$
x_{i}^{\rho}=\sum_{r=1}^{k} n_{r}^{\rho} \Phi_{i}^{H}
$$

while by 1.3 we have

2. 2

$$
\Phi_{i}^{H}=\sum_{\rho} \operatorname{m}_{t}^{\rho} x_{i}^{\rho}
$$

If we substitute 2.2 in 2.1 , we have

$$
\Sigma m_{\rho}^{\rho} n_{s}^{\rho}=\delta t_{t s} \quad \text { and } \quad \sum_{t=1}^{k} m_{t}^{\rho} n_{t}^{\sigma}=\delta^{\rho, \sigma}
$$

from which it follows that the matrix $N=\left(n_{s}{ }^{\sigma}\right)$ is the inverse of $M=\left(m_{s}^{p}\right)$, and so non-singular. Now, from 1.2 , it is sufficient to observe that

$$
\begin{gathered}
\frac{f^{\rho}}{g} \sum_{t=1}^{k} n_{t}^{\rho} \frac{\Sigma^{i} P^{H_{t}}}{h_{t}}=\frac{f^{\rho}}{g} \sum_{\sigma} m_{t}^{\sigma} s^{\sigma} \text { (by Theorem 1) } \\
=\frac{f^{\rho}}{g} S_{\sigma}^{\sigma} \sum_{t=1}^{k} m_{t}^{\sigma} n_{t}^{\rho} \\
=\frac{f^{\rho}}{g} \cdot S^{\rho}=L^{\rho},
\end{gathered}
$$

which proves the theorem.

If we substitute in the equation satisfied by the primitive idempotents 


$$
\sum_{\rho} L^{\rho}=I,
$$

we have

$$
\sum_{\rho} \frac{f^{\rho}}{g} \sum_{t=1}^{k} n_{t}^{\rho} \frac{\sum P^{H}}{h_{t}}=I,
$$

or

$$
\sum_{\rho} \frac{f^{\rho}}{g} \sum_{t=1}^{k} n_{t}^{\rho} \sum_{i=1}^{k} \Phi_{i}^{H} C_{i}=I
$$

By equating coefficients of the classes, we obtain

$$
\sum_{\rho} \frac{f^{\rho}}{g} \sum_{t=1}^{k} n_{t}^{\rho} f_{t}=1
$$

and

$$
\sum_{\rho} f_{t=1}^{\rho} \sum_{t}^{k} f_{t}^{\rho} g_{i}^{H}=0 \text { for } i>1
$$

where $f_{t}$ is the degree of the permutation representation $G^{H}$.

Example 2. As an illustration of the above ideas, let us calculate the matrices $\mathrm{N}$ in the case of $\mathrm{S}_{3}$. This grouphas the following four subgroups:

$$
\begin{aligned}
& \mathrm{H}_{1}=\mathrm{I} \\
& \mathrm{H}_{2}=I,(12) \\
& \mathrm{H}_{3}=I,(123),(132) \\
& \mathrm{H}_{4}=I,(12),(13),(23),(123),(132) .
\end{aligned}
$$


Any three of the se subgroups taken together satisfy the required conditions of Theorem 3 . If $\mathrm{N}_{r, s, t}$ denotes the matrix corresponding to the subgroups $\mathrm{H}_{r}, \mathrm{H}_{\mathrm{s}}, \mathrm{H}_{\mathrm{t}}$, then we have

$\mathrm{N}_{1,2,3}=\left[\begin{array}{rrr}\frac{1}{2} & -1 & \frac{1}{2} \\ \frac{1}{2} & 0 & -\frac{1}{2} \\ -\frac{1}{2} & 1 & \frac{1}{2}\end{array}\right], \quad \mathrm{N}_{1,2,4}=\left[\begin{array}{ccc}1 & -2 & 1 \\ 0 & 1 & -1 \\ 0 & 0 & 1\end{array}\right]$

$\mathrm{N}_{2,3,4}=\left[\begin{array}{ccc}0 & 1 & -1 \\ 1 & 0 & -1 \\ 0 & 0 & 1\end{array}\right], \quad \mathrm{N}_{1,3,4}=\left[\begin{array}{ccc}0 & 1 & -1 \\ \frac{1}{2} & -\frac{1}{2} & 0 \\ 0 & 0 & 1\end{array}\right]$

and $\rho_{1}=\left[1^{3}\right], \rho_{2}=[2,1], \rho_{3}=[3]$.

In order to construct the idempotent $L^{[2,1]}$, consider any set of subgroups, say $\mathrm{H}_{1}, \mathrm{H}_{2}, \mathrm{H}_{3} \cdot$ Their $\Sigma^{\prime}$ sums are respectively

$$
\begin{aligned}
& \Sigma \cdot P^{\mathrm{H}^{1}}=6 \mathrm{I} \\
& \Sigma \cdot P^{\mathrm{H}_{2}}=6 \mathrm{I}+2 \mathrm{C}_{2} \\
& \Sigma \cdot P^{\mathrm{H}_{2}}=6 \mathrm{I}+6 \mathrm{C}_{3},
\end{aligned}
$$

where $C_{2}=(12)+(13)+(23)$ and $C_{3}=(123)+(132)$. Then 


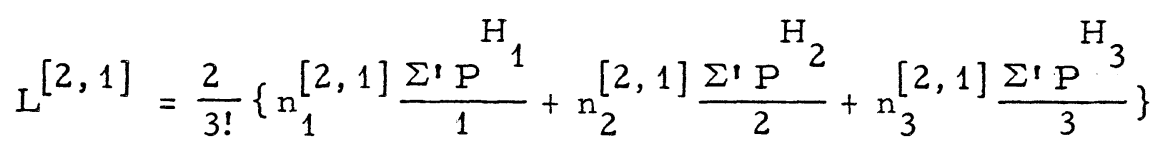

$$
\begin{aligned}
& =\frac{2}{3 !}\left\{\frac{1}{2} \cdot 6 I+0 \frac{\left(6 I+2 C_{2}\right)}{2}-\frac{1}{2} \frac{\left(6 I+6 C_{3}\right)}{3}\right\} \\
& =\frac{2}{3 !}\left\{2 I-C_{3}\right\} \text {, }
\end{aligned}
$$

which is the same as that given by Young's method [3].

\section{REFERENCES}

1. R. Brauer, A characterization of characters of finite groups, Annals of Mathematics, Vol. 57, No. 2, (1953).

2. W. Prokop, Thesis, Eidgenössische Technische Hochscule, Zurich (1948).

3. G. de B. Robinson, The Representation Theory of the Symmetric Group, University of Toronto Press (1961).

University of Toronto

and

University of Mysore 